\title{
Prospective study of myelin water fraction changes after mild traumatic brain injury in collegiate contact sports
}

\author{
Heather S. Spader, MD, ${ }^{1}$ Douglas C. Dean III, PhD, ${ }^{2}$ W. Curt LaFrance Jr., MD, MPH, ${ }^{3-5}$ \\ Neha P. Raukar, MD, MS, ${ }^{6}$ G. Rees Cosgrove, MD, FRCSC, ${ }^{10}$ Stephanie A. Eyerly-Webb, PhD, ${ }^{8}$ \\ Anna Ellermeier, MD, ${ }^{7}$ Stephen Correia, PhD, ${ }^{4,9}$ Sean C. L. Deoni, PhD, ${ }^{11,12}$ and Jeffrey Rogg, MD \\ 1Division of Pediatric Neurosurgery, Joe DiMaggio Children's Hospital, and ${ }^{8}$ Office of Human Research, Memorial Healthcare \\ System, Hollywood, Florida; ${ }^{2}$ Waisman Center, University of Wisconsin-Madison, Wisconsin; ${ }^{3}$ Division of Neuropsychiatry and \\ Behavioral Neurology, ${ }^{6}$ Department of Emergency Medicine, and ${ }^{7}$ Department of Diagnostic Imaging, Rhode Island Hospital; \\ ${ }^{4}$ Department of Psychiatry and Human Behavior, ${ }^{5}$ Department of Neurology, and ${ }^{11}$ Advanced Baby Imaging Lab, School of \\ Engineering, Brown University; and ${ }^{9}$ Providence VA Medical Center, Providence; and ${ }^{12}$ Department of Pediatrics, Memorial \\ Hospital of Rhode Island, Pawtucket, Rhode Island; and ${ }^{10}$ Department of Neurosurgery, Brigham and Women's Hospital, Harvard \\ Medical School, Boston, Massachusetts
}

OBJECTIVE Mild traumatic brain injury (mTBI) in athletes, including concussion, is increasingly being found to have long-term sequelae. Current imaging techniques have not been able to identify early damage caused by mTBI that is predictive of long-term symptoms or chronic traumatic encephalopathy. In this preliminary feasibility study, the authors investigated the use of an emerging magnetic resonance imaging (MRI) technique, multicomponent driven equilibrium single pulse observation of T1 and T2 (mcDESPOT), in visualizing acute and chronic white matter changes after mTBI in collegiate football and rugby players.

METHODS This study was a nonrandomized, nonblinded prospective trial designed to quantify changes in the myelin water fraction (MWF), used as a surrogate MRI measure of myelin content, in a group of male collegiate football and rugby players, classified here as a contact sport player (CSP) cohort, at the time of mTBI diagnosis and 3 months after injury when the acute symptoms of the injury had resolved. In addition, differences in the MWF between the CSP cohort and a control cohort of noncontact sport players (NCSPs) were quantified. T-tests and a threshold-free cluster enhancement (TFCE) statistical analysis technique were used to identify brain structures with significant changes in the MWF between the CSP and NCSP cohorts and between immediately postinjury and follow-up images obtained in the CSP cohort.

RESULTS Brain MR images of 12 right-handed male CSPs were analyzed and compared with brain images of 10 righthanded male NCSPs from the same institution. A comparison of CSP and NCSP baseline images using TFCE showed significantly higher MWFs in the bilateral basal ganglia, anterior and posterior corpora callosa, left corticospinal tract, and left anterior and superior temporal lobe $(p<0.05)$. At the 3 -month follow-up examination, images from the CSP cohort still showed significantly higher MWFs than those identified on baseline images from the NCSP cohort in the bilateral basal ganglia, anterior and posterior corpora callosa, and left anterior temporal lobe, and also in the bilateral corticospinal tracts, parahippocampal gyrus, and bilateral juxtapositional (previously known as supplemental motor) areas $(p<0.05)$. In the CSP cohort, a t-test comparing the MWF at the time of injury and 3 months later showed a significant increase in the overall MWF at follow-up $(p<0.005)$. These increases were greatest in the bilateral basal ganglia and deep white matter. MWF decreases were seen in more superficial white matter $(p<0.005)$.

CONCLUSIONS In this preliminary study, MWF was found to be increased in the brains of CSPs compared with the brains of controls, suggesting acute/chronic MWF alterations in CSPs from previous injuries. Increases in the MWF were

\footnotetext{
ABBREVIATIONS ASL = arterial spin labeling; CSP = contact sport player; $C T E=$ chronic traumatic encephalopathy; $D T I=$ diffusion tensor imaging; FA = fractional anisotropy; FLAIR = fluid-attenuated inversion recovery; FWE = familywise error; IR-SPGR = inversion recovery-spoiled gradient recalled echo sequence; mcDESPOT = multicomponent driven equilibrium single pulse observation of T1 and T2; MCR = multicomponent relaxation; MP-RAGE = magnetization prepared rapid acquisition gradient echo; $\mathrm{mTBI}=$ mild traumatic brain injury; MWF = myelin water fraction; NCSP = non-CSP; SSFP = steady-state free precession; SWI = susceptibility weighted imaging; TFCE $=$ threshold-free cluster enhancement.
}

SUBMITTED July 6, 2017. ACCEPTED December 5, 2017.

INCLUDE WHEN CITING Published online May 1, 2018; DOI: 10.3171/2017.12.JNS171597. 
also demonstrated in the brains of CSPs 3 months after the players sustained an mTBI. The full clinical significance of an increased MWF and whether this reflects axon neuropathology or disorderly remyelination leading to hypermyelination has yet to be determined.

https://thejns.org/doi/abs/10.3171/2017.12.JNS171597

KEYWORDS mild traumatic brain injury; concussion; sports; myelin

$\mathrm{T}$ Raumatic brain injury affects an estimated 1.7 million people in the United States annually, ${ }^{8}$ with approximately $75 \%$ of these injuries categorized as mild. Football is responsible for one of the highest frequencies of mild traumatic brain injury (mTBI) in athletics; approximately 5\% of high school and collegiate football players are diagnosed with an mTBI annually. ${ }^{20}$ Approximately $20 \%$ of these athletes will have disabling symptoms beyond 3 months postinjury. ${ }^{37}$ Repeated mTBIs have also been recently linked to chronic traumatic encephalopathy (CTE), a debilitating neurodegenerative condition that develops later in life. ${ }^{6,39}$

These findings emphasize the need for better diagnostic and prognostic tools for mTBI, especially in populations at risk for repeated mTBIs. The lack of highly sensitive clinical neuroimaging and neuropsychiatric markers of subtle changes due to mTBI makes it difficult to characterize injury severity and to predict outcomes. Researchers are currently investigating new methods for assessing signs of permanent or destructive injuries in young athletes with mTBI. Multiple studies using quantitative MRI analysis have shown decreased cortical volumes after mTBI, from which we can infer decreases in either white or gray matter. ${ }^{40}$

White matter tracts in the brain, both myelinated and nonmyelinated, are susceptible to damage from the impact-acceleration forces experienced during a TBI. Blast impacts cause linear and rotational acceleration of the brain, and the resulting shear strains can severely damage axons (stretch, sever) and their myelin sheaths (separate, decompensate, fragment). ${ }^{2,26,45}$ It has been reported that myelin is particularly vulnerable to secondary damage (decompaction, demyelination) as a result of secondary chemical cascades and neuroinflammation that occur acutely and chronically after a TBI.,26,45 Compromised myelin has been shown to cause reciprocal damage to the underlying axon, and further myelin debris can inhibit the differentiation of progenitor cells that are required for remyelination and axon repair. ${ }^{2,26,45} \mathrm{In}$ all, there is evidence that significant white matter injury and myelin loss occur in mTBI, and this damage may be chronic and can negatively affect neural processing speed and cognitive function. $2,26,45$

The neuroimaging of white matter is of particular interest, therefore, for assessing mTBI and TBI. ${ }^{26,45}$ Diffusion tensor imaging (DTI) characterizes the underlying microstructure and architecture of white matter through measurements of anisotropy and has thus far shown promise as a tool for approximating injuries to the axon and myelin sheaths ${ }^{40}$ Multiple studies focusing on mTBI have shown decreased fractional anisotropy (FA) values in white matter tracts, suggesting tract damage and, potentially, decreased myelin. $1,5,21,23,29,30,34,38$ Furthermore, researchers have correlated decreased FA values with worsened neuropsychiatric outcomes. ${ }^{5}$ Alternatively, multicomponent relaxation (MCR) MRI may be used to assess myelin content in the human brain. ${ }^{15}$ In particular, an emerging MCR technique, known as multicomponent driven equilibrium single pulse observation of T1 and T2 (mcDESPOT), may provide improved insights into the study of myelination after mTBI. The basis of mcDESPOT relies on the knowledge that distinct water compartments exist within neural tissue and that the longitudinal (T1) and transverse (T2) relaxation times associated with these water pools can be characterized by modeling the acquired MRI signal into subvoxel contributions. Within the mcDESPOT model, the myelin water fraction (MWF), a surrogate MRI measure of myelin content, ${ }^{32}$ is the volume fraction associated with the myelin water pool. Specifically, it provides a voxel-wise estimation ranging from 0 to 1 for myelin content, with higher values providing an indirect marker of greater myelin integrity. The MWF derived from this technique has been shown to correlate strongly with histological findings of myelin density ${ }^{22}$ and to provide strong evidence of the technique's sensitivity to myelination through studies of neurodevelopment ${ }^{11,12,16,35}$ and neurological disease. ${ }^{10,13,27,43}$ In addition, the reliability of using a MWF control population has been discussed in other reports of mcDESPOT studies in which multiple sclerosis, amyotrophic lateral sclerosis, and mTBI were examined. ${ }^{4,9,28}$ Unlike DTI, which primarily gives information about myelin-axon bundle interactions, mcDESPOT provides quantitative values with a high sensitivity to myelin on a voxel level. ${ }^{18}$

In this study, our aim was to collect preliminary data on the feasibility of assessing myelin alterations associated with mTBI by using MWF data obtained from the mcDESPOT imaging technique. In a cohort of male collegiate contact sport players (CSPs), we quantified changes in MWF at the time of mTBI diagnosis and again 3 months after injury, when the patients' acute symptoms had resolved. In addition, images of MWFs in the brains of these CSPs were compared with images of MWFs in the brains of a control population of noncontact sport players (NCSPs) to describe potential differences that may suggest acute/chronic white matter remodeling in the contact sport athletes.

\section{Methods Study Design}

A total of 31 patients were enrolled in this study: 21 CSPs and 10 NCSPs. Consent was obtained in accordance with the Brown University Institutional Review Board (protocol \#262023). Exclusion criteria included a history of psychiatric or other neurological disorder, or a contra- 
indication to the performance of an MRI examination. Individuals whose mTBI was diagnosed more than 72 hours after head impact were also excluded.

Football players and rugby players were candidates for the CSP cohort. The football players were part of a Division I NCAA team (Brown University; Providence, RI). At the university, rugby is classified as a club sport. All patients were diagnosed with a practice-related or gamerelated mTBI (Glasgow Coma Scale scores 13-15), as determined by the team athletic trainer or team physician. MRI was performed within 72 hours after injury (acute phase) and repeated 3 months later (follow-up). Athletes from the fencing, cross country, and swimming teams at the same institution, who had been recruited via campus flyers, constituted the control group.

Neuropsychiatric data were also acquired at the time of injury and at the 3-month follow-up examination by using the following tests: imPACT Testing (which also included baseline scores), Beck Depression Inventory, Beck Anxiety Inventory, Symptom Checklist 90, and Ten-Item Personality Inventory.

\section{Myelin Water Imaging and Processing \\ Image Acquisition}

All patients underwent imaging at the Brown University MRI Research Facility on the same Siemens 3T Tim Trio MRI unit with a 12-channel head coil. For the mcDESPOT acquisition, a series of T1-weighted spoiled gradient recalled echo sequences (SPGR; or spoiled fluid-attenuated inversion recovery [FLASH]) and T1/T2weighted balanced steady-state free precession (SSFP) images were acquired over a range of flip angles. In addition, an inversion recovery SPGR (IR-SPGR) sequence was acquired to correct for transmit magnetic field inhomogeneities. ${ }^{14}$ Whole-brain imaging data were collected using the following parameters: field of view $220 \times 220 \times$ $176 \mathrm{~mm}$ and image matrix $112 \times 112 \times 88$; combined, this resulted in approximately $1.8 \times 1.8 \times 1.8 \mathrm{~mm}^{3}$ isotropic voxel resolution. The total mcDESPOT acquisition time for each patient was less than 12 minutes. A fully detailed description of mcDESPOT parameters is available elsewhere. ${ }^{16}$

During the imaging session, additional sequences were also acquired, including magnetization prepared rapid acquisition gradient echo (MP-RAGE), susceptibility weighted imaging (SWI), arterial spin labeling (ASL), FLAIR, and DTI (30-direction acquisition). The total imaging time for all sequences for each participant was 60 minutes.

\section{Generation of MWF Maps}

McDESPOT sequences (SPGR, IR-SPGR, and balanced SSFP) were linearly coregistered to account for head movement, ${ }^{24}$ and nonparenchymal voxels were removed. ${ }^{41}$ From these data, $\mathrm{B}_{0}$ and $\mathrm{B}_{1}$ field calibration maps were calculated..$^{14}$ Last, the MWF maps were calculated for each voxel by fitting the SPGR and balanced SSFP data to a 3 -pool tissue model that estimates fractional volumes and relaxation times for intra- and extra-axonal water, myelinassociated water, and nonexchanging free water. ${ }^{17}$ This 3-pool model was designed for use with pathological conditions in which there may be edema that could affect the calculation of the MWF map. Specifically, the inclusion of a nonexchanging water pool helps adequately control for the effects of slow relaxing water, as would be expected with edema. This model has been shown to provide robust and reproducible findings through numerical simulation and in vivo data. ${ }^{12}$

MWF maps were nonlinearly normalized into a common analysis space, allowing for comparisons across patients and controls. A T1-weighted template was used as the reference space to which MWF maps of the current study were registered. ${ }^{16}$ The high flip angle SPGR image was normalized to the T1-weighted template using symmetric diffeomorphic normalization to determine the transformations necessary to map an individual's raw data space to the template space. ${ }^{3}$ These resulting transformations were applied to each individual's MWF map, aligning the MWF map to the reference template. Full details regarding image normalization are described elsewhere. ${ }^{16}$

Final processed images obtained at the 3-month imaging examination show an anatomical white matter mask overlaid with areas of increased (red) or decreased (blue) MWF from baseline.

\section{Statistical Analysis}

Changes in the MWF were evaluated between each CSP's immediate postinjury images and the follow-up image obtained 3 months later. In addition, MWF maps obtained from the NCSP control group were compared with MWF maps from the CSP cohort. A voxel-wise ttest was performed using the randomize tool in the FSL software library version 5.0 (https://fsl.fmrib.ox.ac.uk/fsl/ fslwiki/FSL). ${ }^{42,46}$ Five thousand permutations with and without threshold-free cluster enhancement (TFCE) were used to identify statistically significant differences in the MWF between the time of injury and 3 months afterward, as well as to evaluate the differences between the CSP and NCSP samples. Statistical significance was defined as $p$ $<0.05$ corrected for multiple comparisons using TFCE. In some instances in which analyses did not survive the more rigorous TFCE correction, uncorrected $\mathrm{p}$ values at the higher threshold of $p<0.005$ are also reported. This higher threshold was used to dissuade possible false-positive findings, as these data were not corrected for multiple comparisons. The locations of significant voxels were identified using a white matter-specific atlas described in detail elsewhere. ${ }^{16}$

\section{Results \\ Study Cohorts}

Twenty right-handed, male football players and 1 righthanded, male rugby player who sustained mTBIs comprised the CSP cohort. Of the 21 patients who originally underwent imaging studies, 12 (1 rugby and 11 football players) returned for a 3-month follow-up imaging examination. Therefore, the final CSP cohort used for the analysis consisted of 12 players in whom MRI results obtained at the time of injury and 3 months later were both available. The mean age of the CSP cohort was $20.18 \pm 1.18$ 


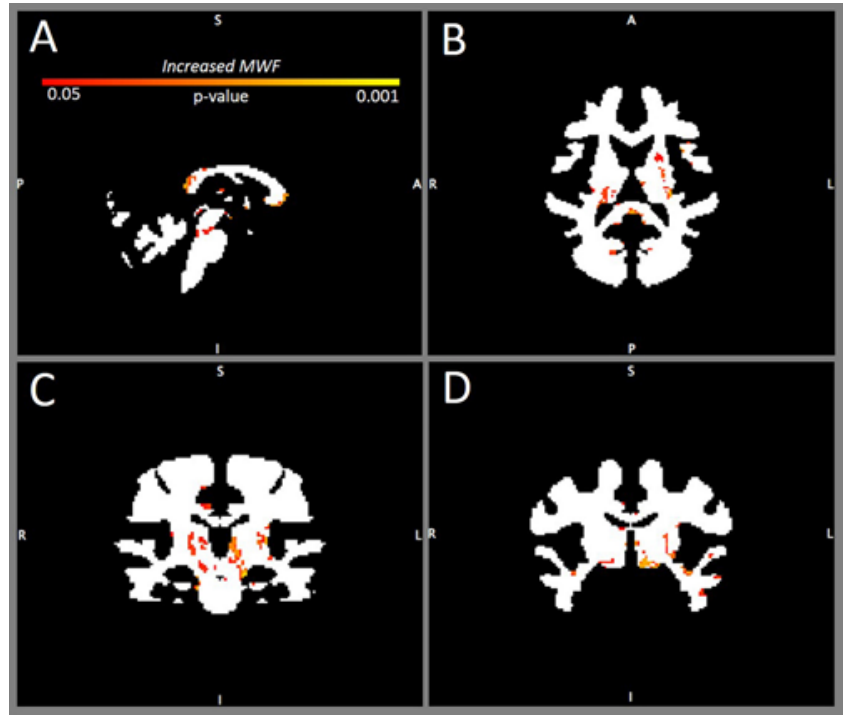

FIG. 1. MWF maps comparing the brains of CSPs at the time of injury with the NCSP baseline. Sagittal (A), axial (B), and coronal (C) MWF maps showing areas of increased MWF ( $p<0.05$, FWE corrected) in the anterior and posterior corpora callosa; bilateral thalami, particularly the anterior thalamic radiations (left > right side); and corticospinal tracts (left > right side). Coronal (D) MWF map demonstrating increased MWF in the left anterior and superior temporal lobe ( $p<0.05$, FWE corrected).

years. One participant acknowledged having sustained a previous concussion, whereas all others had no notable medical history of concussion.

Ten right-handed, male athletes comprised the NCSP cohort. The mean age of the control group was $20.16 \pm$
1.27 years. There was no medical history of concussion in the NCSP cohort.

\section{Comparison of CSP Acute-Phase MRI and NCSP Baseline MRI}

A comparison of MR images obtained in the NCSP control population with acute-phase MR images obtained in the CSP cohort (within 72 hours of injury) showed there was a significantly higher MWF signal in the CSP population ( $\mathrm{p}<0.05$, familywise error [FWE]-corrected) (Fig. $1 \mathrm{~A}-\mathrm{C})$. In particular, the MWF in the CSP group was higher in the anterior and posterior corpora callosa, bilateral thalami (particularly the anterior thalamic radiations [left $>$ right]), corticospinal tracts (left $>$ right), and left anterior and superior temporal lobe (Fig. 1D). There were no areas in which the MWF in the CSP cohort was lower than that in the NCSP population.

\section{Comparison of CSP Follow-Up MRI and NCSP Baseline MRI}

The comparison between images obtained in the control group and follow-up MR images obtained in the CSP cohort 3 months after injury also showed a globally higher MWF in the CSP cohort. A greater MWF was observed mostly in the anterior and posterior corpora callosa, bilateral corticospinal tracts, and thalami (left $>$ right) (Fig. 2A). These MWF increases were seen in the left anterior temporal and parahippocampal gyri (Fig. 2B) as well as in the bilateral juxtapositional lobes $(\mathrm{p}<0.05$, FWE-corrected). As with the acute-phase MR images, there were no areas in which the MWF in the CSP cohort (12 players) was lower than that in the NCSP population.

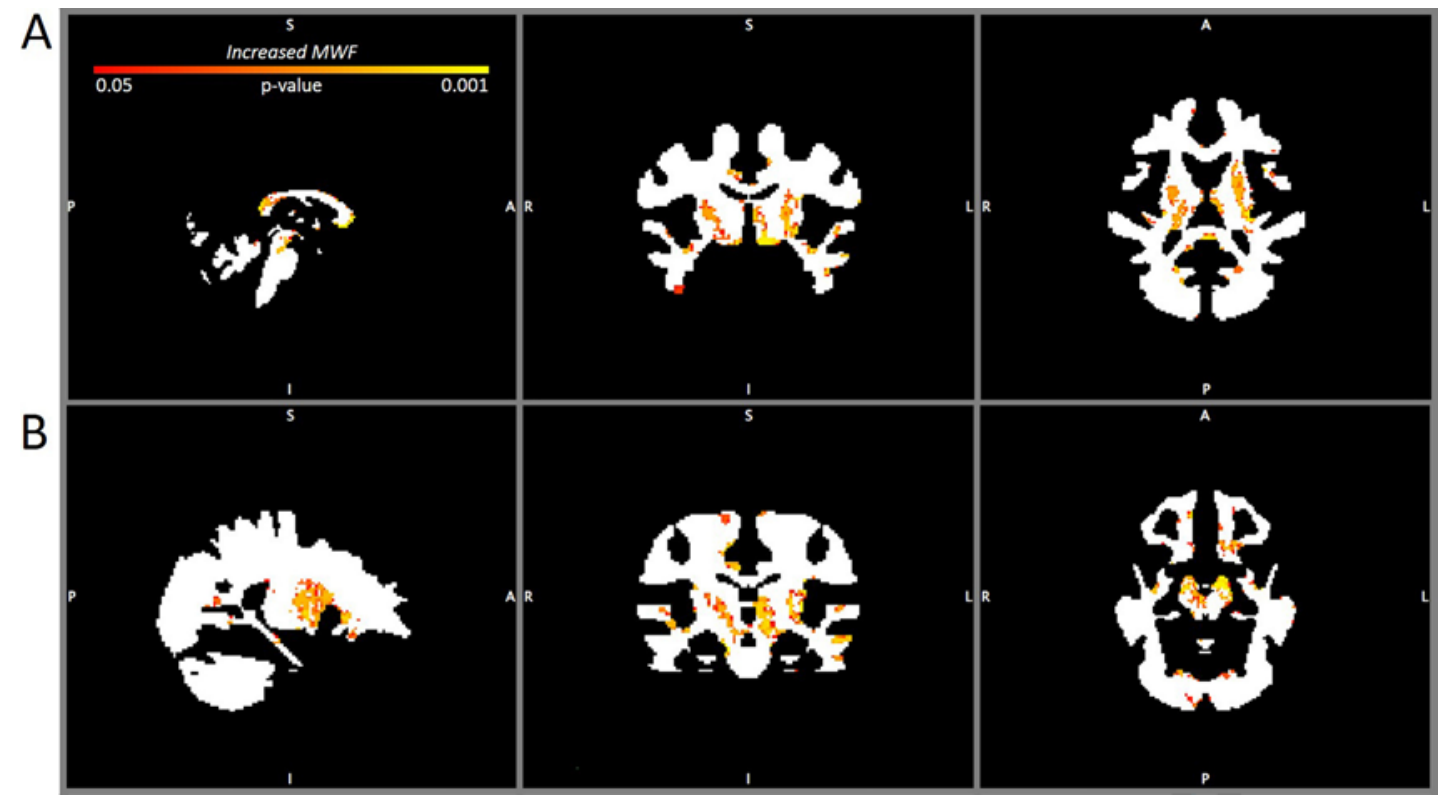

FIG. 2. MWF maps comparing the brains of CSPs 3 months after injury with the NCSP baseline. A: Sagittal (left), coronal (center), and axial (right) MWF maps at the level of the thalamus showing increased MWF ( $p<0.05$, FWE corrected) in the anterior and posterior corpora callosa, thalamus, and midbrain. B: Sagittal (left), coronal (center), and axial (right) MWF maps showed significant MWF increases ( $p<0.05$, FWE corrected) in the midbrain and temporal lobes (left $>$ right temporal lobe). 


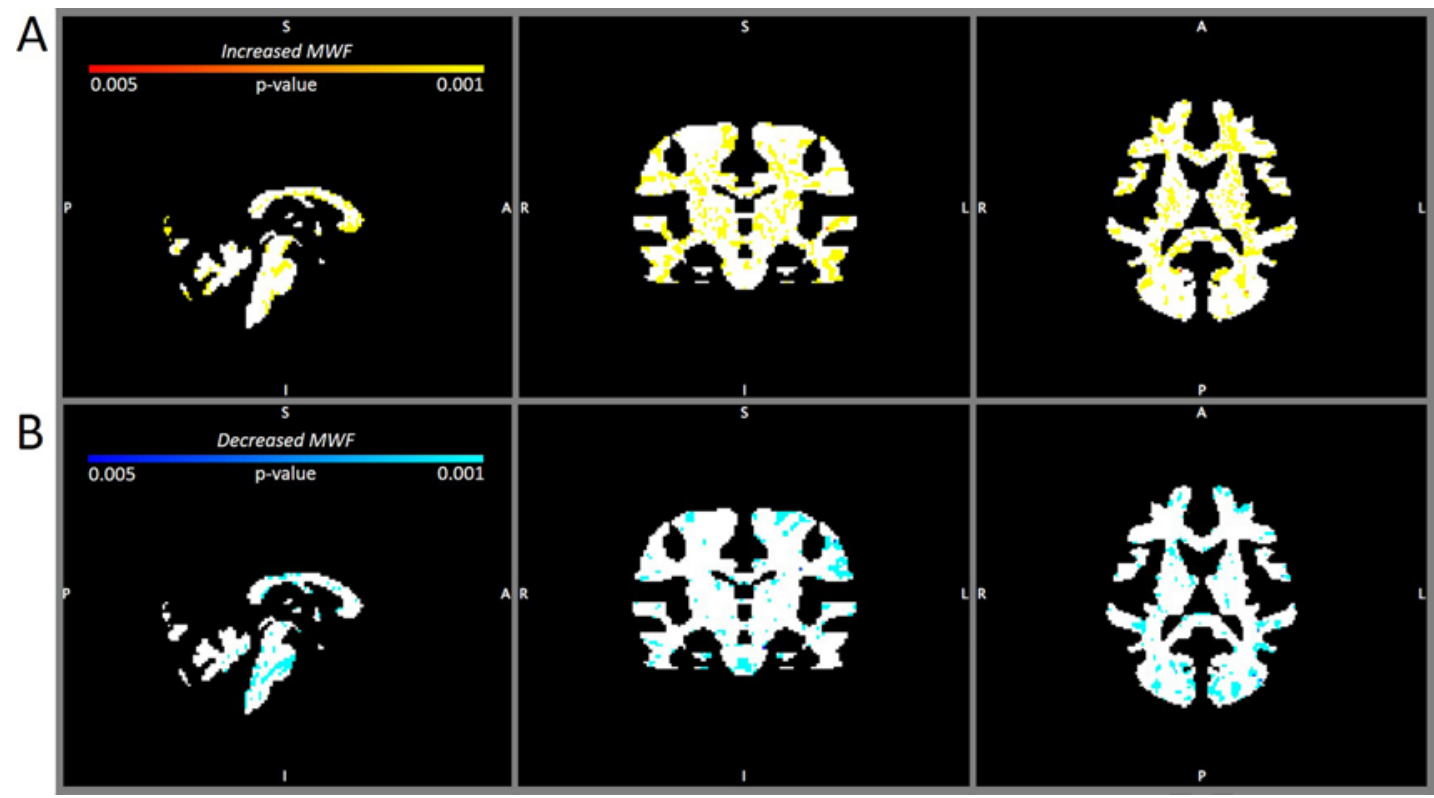

FIG. 3. MWF maps comparing the brains of CSPs at the time of injury and 3 months postinjury. A: Sagittal (left), coronal (center), and axial (right) MWF maps at the level of the thalamus showing areas of increased MWF (yellow). The largest increases occurred in the deep white matter, particularly the bilateral basal ganglia. Increases $(p<0.005$, uncorrected) are also seen in the anterior corpus callosum, bilateral corona radiata, and left temporal lobe. B: Sagittal (left), coronal (center), and axial (right) MWF maps demonstrating areas of decreased MWF (blue) scattered in peripheral white matter ( $p<0.005$, uncorrected).

\section{Comparison of CSP Acute-Phase MRI and CSP 3-Month Follow-Up MRI}

A paired voxel-wise t-test comparing MWF maps obtained during the acute phase of mTBI (within 72 hours) and the 3-month follow-up examination showed a predominant increase in the MWF at the 3-month follow-up (Fig. 3A). The largest MWF increases occurred in the deep white matter. Increases were also seen in the anterior corpus callosum, bilateral corona radiata, superior longitudinal fasciculi, thalami, and left temporal lobe ( $\mathrm{p}<$ 0.005 , uncorrected). Scattered MWF decreases were seen in the more peripheral white matter and bilaterally in the internal capsules (left $>$ right) $(\mathrm{p}<0.005$, uncorrected) (Fig. 3B). MWF changes in the hemispheres, superior longitudinal fasciculi, temporal lobes, corona radiata, and internal capsules are shown as a box plot in Fig. 4. Notably, despite significant findings at an uncorrected threshold of $p<0.005$, the findings from this statistical analysis did not carry through to the more rigorous multiple comparison analysis (TFCE).

\section{Discussion}

In this preliminary study, acute and follow-up myelin neuroimaging results for collegiate athletes with mTBI were compared and evaluated against a control population of noninjured athletes. Only one athlete, a member of the CSP cohort, had previously been diagnosed with an mTBI. The most significant and unique findings of this preliminary study were as follows: 1) a significantly higher MWF was identified in acute-phase MR images obtained in the CSPs than that in images collected from the NCSPs; 2) a significantly higher MWF was identified on follow-up images obtained in the CSPs than that in images collected from the NCSPs; and 3) in CSPs who had suffered mTBI there was an increase in the MWF between that measured on acute-phase MR images (within 72 hours of injury) and that found on follow-up images ( 3 months postinjury).

\section{mTBI and DTI}

MRI studies using DTI in patients with mTBI have found decreases in FA values that are thought to correlate with decreases in white matter integrity, which is often interpreted as decreased myelin..$^{29}$ These post-mTBI changes have been demonstrated most often in the frontal association pathways, which include the anterior corona radiata, uncinate fasciculus, superior longitudinal fasciculus, and anterior corpus callosum. ${ }^{48}$ These FA value decreases have also been found to correlate with persistent cognitive deficits. ${ }^{34,44}$ Although the majority of DTI studies of mTBI have found decreased FA values, particularly in the corpus callosum, internal capsule, centrum semiovale, and other major white matter pathways, some studies have shown increased FA values and decreased mean diffusivity values. ${ }^{40}$ This variability in DTI findings suggests that DTI changes are likely multifactorial and may be due to the timing of imaging versus the time of injury. ${ }^{18}$

\section{Preclinical Studies of Myelin Remodeling After TBI}

Preclinical research has shown that there are white matter changes after a head injury that cannot be seen with conventional neuroimaging. ${ }^{33}$ In rodent models of mTBI, a pattern of cell death, demyelination, and remyelination has 

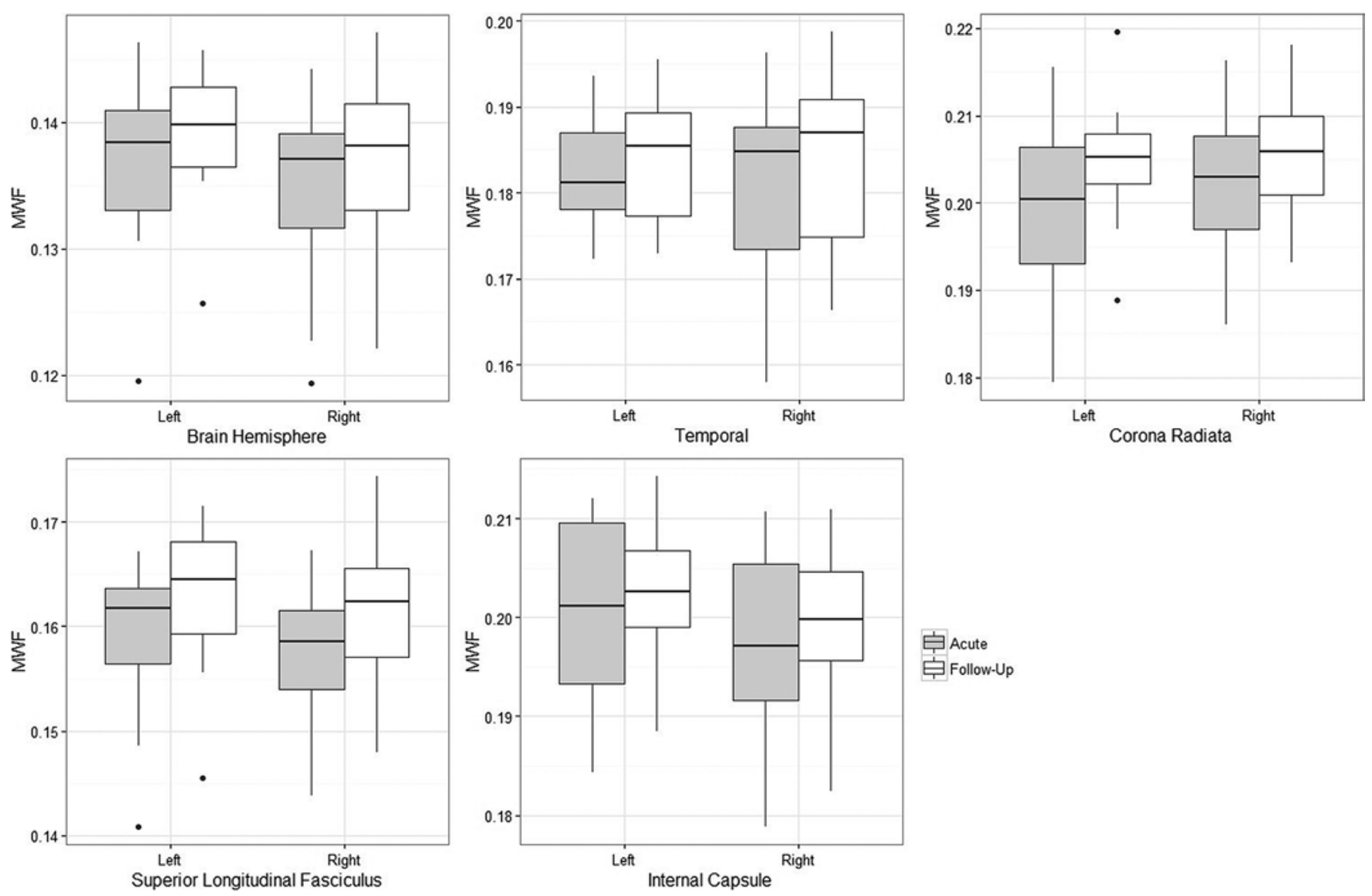

FIG. 4. Box plots of the MWF at the time of acute-phase imaging (gray) and follow-up imaging (white) for the left and right hemispheric regions of multiple brain structures. The box plots show the third quartile, median, and first quartile range of the data; the whiskers represent minimum and maximum values; and data outliers are represented as individual points.

been demonstrated ${ }^{2,26,33,45}$; however, the remyelination seen in these models does not seem to have the same organization as preinjury myelin. In these models, the myelination is redundant, resulting in increased myelin but fewer and smaller axons. ${ }^{7,31,33,36}$ Since these changes are not visible when employing commonly used imaging techniques, the neuropsychiatric, functional, and long-term structural outcomes from these remodeling processes are still unknown. The evidence of disordered remyelination may be part of the explanation for why all CSPs in this study had increased MWFs when compared with the NCSPs.

A similar explanation of disordered myelination may serve to explain DTI studies showing decreased FA values. DTI is not a quantitative myelin imaging technique, and although it provides information about the axon milieu, it does not specifically address the amount of myelin in the myelin sheath. ${ }^{25}$ Therefore, decreases in DTI FA values after mTBI are not in conflict with MWF increases. This can be seen in animal models of mTBI that demonstrate vacuolated myelin regions, abnormally thick myelin sheaths, and small axons. If these findings were imaged, this disorganization would likely result in decreased anisotropy (decreased FA) and increased overall myelin due to increased myelin, albeit disorganized. ${ }^{36}$ The ability to characterize the differences between beneficial myelin biogenesis and disorderly remyelination with neuroimaging could have significant implications for quantifying TBI severity and prognosis.

In a similar study of the MWF in collegiate hockey players, changes in the MWF were also found. In a study by Wright et al., ${ }^{47}$ all participants underwent baseline MRI as well as 72-hour, 2-week, and 2-month post-mTBI follow-up imaging. The authors noted an initial decrease in the MWF followed by a recovery to postinjury myelin levels at the 2-month imaging session, which suggested a similar trajectory of remyelination after injury. No comparison was made to athletes in noncontact sports. ${ }^{47}$ Our study showed that contact sport athletes have increases in the MWF 3 months after mTBI, particularly in the major deep white matter tracts. These increases may be a manifestation of exuberant remyelination following an initial decrease in MWF, as was demonstrated by Wright et al. ${ }^{47}$ Although our CSP cohort may have had an initial acute decrease in the MWF at the time of injury, compared with their preinjury baseline, we could not quantify this because we did not have baseline images.

Changes in the MWF seen in a comparison of NCSPs and CSPs at the time of mTBI and 3 months after the injury are novel findings of this study. Our analysis showed increased myelination in the CSP cohort at the time of in- 

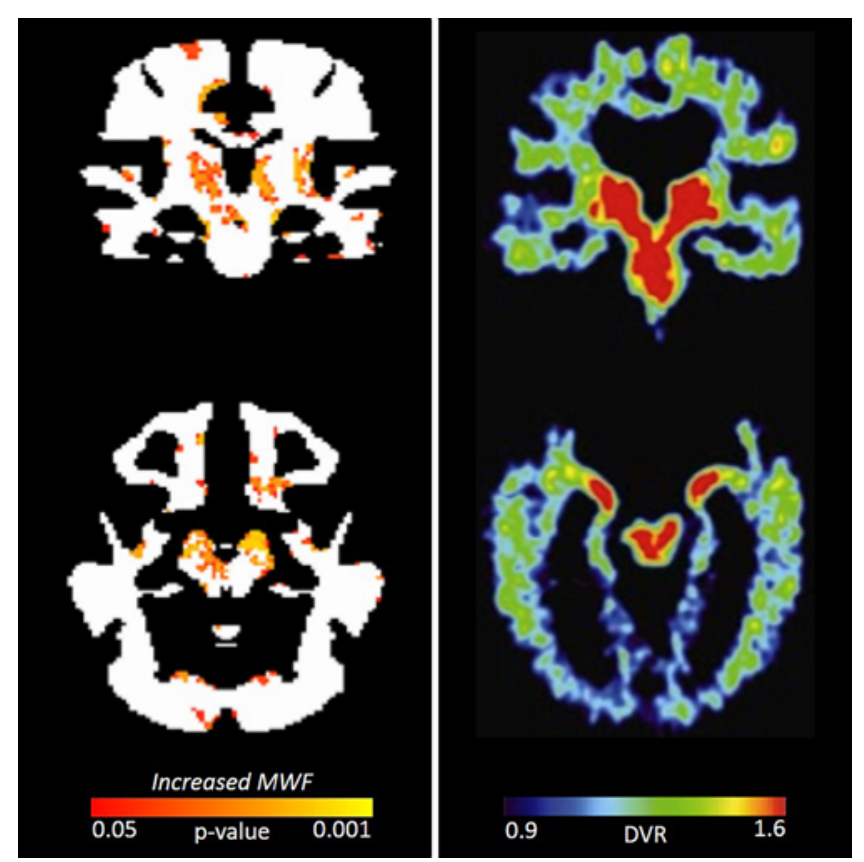

FIG. 5. Comparison of MWF maps obtained in CSPs 3 months after injury with those of NCSPs (left) and with the PET F-18 FDDNP (tausensitive brain imaging agent) signal in retired football players with suspected CTE $^{5}$ (right). Both figures show significant midbrain and thalamic pathology.

jury and even larger increases 3 months afterward. These statistically significant increases in the MWF occurred more on the left than right side of the brain in these righthanded players, which is consistent with previous findings concerning the side of the brain more affected by an mTBI. ${ }^{47}$ The 3-month follow-up MR images also showed increases in the MWF in the bilateral basal ganglia, the rostrum of the corpus callosum, and throughout the entire corticospinal tract.

The preliminary nature of this study limits confidence in ascribing the clinical significance of these novel findings; however, studies of chronic traumatic encephalopathy (CTE) do provide some insight. A PET imaging study of football players with suspected CTE found significant changes in the amygdala, dorsal midbrain, and subcortical and limbic structures on PET scans. These changes were also consistent with autopsy specimens of patients with CTE. ${ }^{4}$ In our study, when the MWF maps of CSPs at injury and 3 months later were compared with MWP maps of NCSP controls, the areas of involvement were similar to those viewed on the PET scans of football players with suspected CTE, with marked similarities in the involvement of the thalamus and midbrain (Fig. 5).

Our results show preliminary evidence that there is a long-term effect on the MWF after mTBI in collegiate athletes who play contact sports and that this effect is visible as an increased MWF correlating with increased myelin. Basic science models indicate that this increased myelin is not beneficial and is likely a result of decreased connectivity, vacuolation, and smaller axons. ${ }^{7,19,31,33,36}$ To corroborate this preliminary evidence of disorganized myelin forma- tion, the MWF needs to be studied in CSPs both before and after an mTBI and compared again with an NCSP population. These studies also need to include DTI data, so that FA values can be compared with MWF values. Future studies also need to incorporate neuropsychiatric testing that shows whether an increased MWF correlates with clinical outcomes. ${ }^{5}$ Although neuropsychiatric data were collected for this study, the population was too small and the test results were too variable to draw any significant statistical correlations between the imaging and neuropsychiatric outcomes.

In summary, mcDESPOT may represent a more direct and robust measure of brain injury than DTI analysis in assessing the extent of microstructural brain injury in athletes who have suffered an mTBI. It may also help identify those individuals who are at greater risk for chronic encephalopathies and other possible mTBI-related injuries later in life. ${ }^{6}$

\section{Limitations}

The primary limitation to this study was the lack of preinjury MR images for athletes who sustained an mTBI to compare as a baseline. Additionally, our sample size of 12 CSPs with acute mTBI and 10 NCSPs was small, but this is not uncommon in prospective, traumatic event-related imaging studies of this nature. Because of this small sample size and the test performance variability, the neuropsychiatric data collected were not robust enough to statistically analyze their association with MWF values. Another limitation is the heterogeneity of potential differences in brain injury. Each individual is likely to be affected differently, and therefore, group analysis methods are only going to be sensitive where changes are occurring across the group.

\section{Conclusions}

In this preliminary investigation, the MWF increased in the brains of CSPs who sustained an MTBI; this was identified both at the 3-month follow-up MRI examination and when compared with a control population of NCSPs. The most significant finding of this study is the increased MWF in the deep white matter tracts in CSPs compared with controls at both time points. These increases may be the result of a reparative process in patients who sustained mTBIs that involves demyelination, remyelination, and even hypermyelination, the significance of which has yet to be defined. This study provides a basis for additional studies aimed at understanding the underlying neuropathophysiology of the brain's recovery from mTBI.

\section{Acknowledgments}

The authors would like to thank Kristin Kraus, MSc, for her editorial assistance. In addition, we would like to thank the Office of Diagnostic Imaging Research at Rhode Island Hospital (Elizabeth Morrell, Wendy Smith, and Sue Foley) for their assistance with this study.

This study was funded by a grant from the Department of Diagnostic Imaging, Rhode Island Hospital, Providence, RI. This work was additionally supported in part by the National Institutes for Mental Health (K99MH110596 to DCD) and the Eunice Kennedy Shriver National Institute of Child Health \& Human Development 
under the National Institutes of Health (T32HD007489 to DCD and U54 HD090256 to the Waisman Center).

\section{References}

1. Arfanakis K, Haughton VM, Carew JD, Rogers BP, Dempsey RJ, Meyerand ME: Diffusion tensor MR imaging in diffuse axonal injury. AJNR Am J Neuroradiol 23:794-802, 2002

2. Armstrong RC, Mierzwa AJ, Marion CM, Sullivan GM: White matter involvement after TBI: Clues to axon and myelin repair capacity. Exp Neurol 275:328-333, 2016

3. Avants BB, Epstein CL, Grossman M, Gee JC: Symmetric diffeomorphic image registration with cross-correlation: evaluating automated labeling of elderly and neurodegenerative brain. Med Image Anal 12:26-41, 2008

4. Barrio JR, Small GW, Wong KP, Huang SC, Liu J, Merrill $\mathrm{DA}$, et al: In vivo characterization of chronic traumatic encephalopathy using [F-18]FDDNP PET brain imaging. Proc Natl Acad Sci U S A 112:E2039-E2047, 2015 (Erratum in Proc Natl Acad Sci U S A 112:E2981, 2015)

5. Bazarian JJ, Zhu T, Blyth B, Borrino A, Zhong J: Subjectspecific changes in brain white matter on diffusion tensor imaging after sports-related concussion. Magn Reson Imaging 30:171-180, 2012

6. Blennow K, Brody DL, Kochanek PM, Levin H, McKee A, Ribbers GM, et al: Traumatic brain injuries. Nat Rev Dis Primers 2:16084, 2016

7. Bramlett HM, Dietrich WD: Quantitative structural changes in white and gray matter 1 year following traumatic brain injury in rats. Acta Neuropathol 103:607-614, 2002

8. Centers for Disease Control and Prevention: Report to Congress. Traumatic Brain Injury in the United States: Epidemiology and Rehabilitation. Atlanta: Centers for Disease Control and Prevention, 2015 (https://www.cdc.gov/ traumaticbraininjury/pdf/tbi_report_to_congress_epi_and_ rehab-a.pdf) [Accessed January 24, 2018]

9. Combes AJE, Matthews L, Lee JS, Li DKB, Carruthers R, Traboulsee AL, et al: Cervical cord myelin water imaging shows degenerative changes over one year in multiple sclerosis but not neuromyelitis optica spectrum disorder. Neuroimage Clin 16:17-22, 2017

10. Dean DC III, Hurley SA, Kecskemeti SR, O'Grady JP, Canda C, Davenport-Sis NJ, et al: Association of amyloid pathology with myelin alteration in preclinical Alzheimer disease. JAMA Neurol 74:41-49, 2017

11. Dean DC III, O'Muircheartaigh J, Dirks H, Travers BG, Adluru N, Alexander AL, et al: Mapping an index of the myelin g-ratio in infants using magnetic resonance imaging. Neuroimage 132:225-237, 2016

12. Dean DC III, O'Muircheartaigh J, Dirks H, Waskiewicz N, Walker L, Doernberg E, et al: Characterizing longitudinal white matter development during early childhood. Brain Struct Funct 220:1921-1933, 2015

13. Dean DC III, Sojkova J, Hurley S, Kecskemeti S, Okonkwo $\mathrm{O}$, Bendlin BB, et al: Alterations of myelin content in Parkinson's disease: a cross-sectional neuroimaging study. PLoS One 11:e0163774, 2016

14. Deoni SC: Correction of main and transmit magnetic field (B0 and B1) inhomogeneity effects in multicomponent-driven equilibrium single-pulse observation of T1 and T2. Magn Reson Med 65:1021-1035, 2011

15. Deoni SC: Quantitative relaxometry of the brain. Top Magn Reson Imaging 21:101-113, 2010

16. Deoni SC, Dean DC III, O'Muircheartaigh J, Dirks H, Jerskey BA: Investigating white matter development in infancy and early childhood using myelin water faction and relaxation time mapping. Neuroimage 63:1038-1053, 2012

17. Deoni SC, Matthews L, Kolind SH: One component? Two components? Three? The effect of including a nonexchanging "free" water component in multicomponent driven equilibrium single pulse observation of T1 and T2. Magn Reson Med 70:147-154, 2013

18. Deoni SC, Rutt BK, Jones DK: Investigating exchange and multicomponent relaxation in fully-balanced steady-state free precession imaging. J Magn Reson Imaging 27:1421-1429, 2008

19. Gao X, Chen J: Mild traumatic brain injury results in extensive neuronal degeneration in the cerebral cortex. J Neuropathol Exp Neurol 70:183-191, 2011

20. Guskiewicz KM, Weaver NL, Padua DA, Garrett WE Jr: Epidemiology of concussion in collegiate and high school football players. Am J Sports Med 28:643-650, 2000

21. Holli KK, Harrison L, Dastidar P, Wäljas M, Liimatainen S, Luukkaala T, et al: Texture analysis of MR images of patients with mild traumatic brain injury. BMC Med Imaging 10:8, 2010

22. Hurley SA, Mossahebi P, Samsonov AA, Alexander AL, Deoni SC, Fisher R, et al: Multicomponent relaxometry (mcDESPOT) in the shaking pup model of dysmyelination, presented at the ISMRM-ESMRMB Joint Annual Meeting, Stockholm, Sweden, 2010 (Poster) (http://dev.ismrm. org/2010/4516.html) [Accessed January 24, 2018]

23. Inglese M, Makani S, Johnson G, Cohen BA, Silver JA, Gonen O, et al: Diffuse axonal injury in mild traumatic brain injury: a diffusion tensor imaging study. J Neurosurg 103:298-303, 2005

24. Jenkinson M, Bannister P, Brady M, Smith S: Improved optimization for the robust and accurate linear registration and motion correction of brain images. Neuroimage 17:825-841, 2002

25. Jones DK, Knösche TR, Turner R: White matter integrity, fiber count, and other fallacies: the do's and don'ts of diffusion MRI. Neuroimage 73:239-254, 2013

26. Jurick SM, Bangen KJ, Evangelista ND, Sanderson-Cimino M, Delano-Wood L, Jak AJ: Advanced neuroimaging to quantify myelin in vivo: application to mild TBI. Brain Inj 30:1452-1457, 2016

27. Kolind S, Matthews L, Johansen-Berg H, Leite MI, Williams SC, Deoni S, et al: Myelin water imaging reflects clinical variability in multiple sclerosis. Neuroimage 60:263-270, 2012

28. Kolind S, Sharma R, Knight S, Johansen-Berg H, Talbot K, Turner MR: Myelin imaging in amyotrophic and primary lateral sclerosis. Amyotroph Lateral Scler Frontotemporal Degener 14:562-573, 2013

29. Kraus MF, Susmaras T, Caughlin BP, Walker CJ, Sweeney JA, Little DM: White matter integrity and cognition in chronic traumatic brain injury: a diffusion tensor imaging study. Brain 130:2508-2519, 2007

30. Lipton ML, Gellella E, Lo C, Gold T, Ardekani BA, Shifteh $\mathrm{K}$, et al: Multifocal white matter ultrastructural abnormalities in mild traumatic brain injury with cognitive disability: a voxel-wise analysis of diffusion tensor imaging. J Neurotrauma 25:1335-1342, 2008

31. Lotocki G, de Rivero Vaccari JP, Alonso O, Molano JS, Nixon R, Safavi P, et al: Oligodendrocyte vulnerability following traumatic brain injury in rats. Neurosci Lett 499:143-148, 2011 (Erratum in Neurosci Lett 516:166, 2012)

32. MacKay A: Magnetic resonance of myelin water: an in vivo marker for myelin. Brain Plast 2:71-91, 2016

33. Mierzwa AJ, Marion CM, Sullivan GM, McDaniel DP, Armstrong RC: Components of myelin damage and repair in the progression of white matter pathology after mild traumatic brain injury. J Neuropathol Exp Neurol 74:218-232, 2015

34. Niogi SN, Mukherjee P, Ghajar J, Johnson C, Kolster RA, Sarkar R, et al: Extent of microstructural white matter injury in postconcussive syndrome correlates with impaired cognitive reaction time: a $3 \mathrm{~T}$ diffusion tensor imaging study 
of mild traumatic brain injury. AJNR Am J Neuroradiol 29:967-973, 2008

35. O'Muircheartaigh J, Dean DC III, Ginestet CE, Walker L, Waskiewicz N, Lehman K, et al: White matter development and early cognition in babies and toddlers. Hum Brain Mapp 35:4475-4487, 2014

36. Rodriguez-Paez AC, Brunschwig JP, Bramlett HM: Light and electron microscopic assessment of progressive atrophy following moderate traumatic brain injury in the rat. Acta Neuropathol 109:603-616, 2005

37. Ruff RM, Camenzuli L, Mueller J: Miserable minority: emotional risk factors that influence the outcome of a mild traumatic brain injury. Brain Inj 10:551-565, 1996

38. Rutgers DR, Fillard P, Paradot G, Tadié M, Lasjaunias P, Ducreux D: Diffusion tensor imaging characteristics of the corpus callosum in mild, moderate, and severe traumatic brain injury. AJNR Am J Neuroradiol 29:1730-1735, 2008

39. Safinia C, Bershad EM, Clark HB, SantaCruz K, Alakbarova N, Suarez JI, et al: Chronic traumatic encephalopathy in athletes involved with high-impact sports. J Vasc Interv Neurol 9:34-48, 2016

40. Shenton ME, Hamoda HM, Schneiderman JS, Bouix S, Pasternak O, Rathi Y, et al: A review of magnetic resonance imaging and diffusion tensor imaging findings in mild traumatic brain injury. Brain Imaging Behav 6:137-192, 2012

41. Smith SM: Fast robust automated brain extraction. Hum Brain Mapp 17:143-155, 2002

42. Smith SM, Jenkinson M, Johansen-Berg H, Rueckert D, Nichols TE, Mackay CE, et al: Tract-based spatial statistics: voxelwise analysis of multi-subject diffusion data. Neuroimage 31:1487-1505, 2006

43. Spader HS, Ellermeier A, O'Muircheartaigh J, Dean DC III, Dirks H, Boxerman JL, et al: Advances in myelin imaging with potential clinical application to pediatric imaging. Neurosurg Focus 34(4):E9, 2013

44. Wada T, Asano Y, Shinoda J: Decreased fractional anisotropy evaluated using tract-based spatial statistics and correlated with cognitive dysfunction in patients with mild traumatic brain injury in the chronic stage. AJNR Am J Neuroradiol 33:2117-2122, 2012

45. Weber AM, Torres C, Rauscher A: Imaging the role of myelin in concussion. Neuroimaging Clin N Am 28:83-90, 2018

46. Winkler AM, Ridgway GR, Webster MA, Smith SM, Nichols TE: Permutation inference for the general linear model. Neuroimage 92:381-397, 2014

47. Wright AD, Jarrett M, Vavasour I, Shahinfard E, Kolind S, van Donkelaar P, et al: Myelin water fraction is transiently reduced after a single mild traumatic brain injury-a prospective cohort study in collegiate hockey players. PLoS One 11:e0150215, 2016

48. Yuh EL, Cooper SR, Mukherjee P, Yue JK, Lingsma HF, Gordon WA, et al: Diffusion tensor imaging for outcome prediction in mild traumatic brain injury: a TRACK-TBI study. J Neurotrauma 31:1457-1477, 2014

\section{Disclosures}

Dr. Deoni reports being a consultant to Nestlé.

\section{Author Contributions}

Conception and design: Spader, LaFrance, Raukar, Cosgrove, Ellermeier, Correia, Deoni, Rogg. Analysis and interpretation of data: Spader, Dean, LaFrance, Cosgrove, Ellermeier, Deoni, Rogg. Drafting the article: Spader, Dean. Critically revising the article: all authors. Reviewed submitted version of manuscript: all authors. Approved the final version of the manuscript on behalf of all authors: Spader. Statistical analysis: Spader, Dean, Deoni.

\section{Correspondence}

Heather Spader: Joe DiMaggio Children's Hospital, Hollywood, FL.hspader@mhs.net. 